\title{
Cancer Chemoprevention by Citrus Pulp and Juices Containing High Amounts of $\beta$-Cryptoxanthin and Hesperidin
}

\author{
Takuji Tanaka, ${ }^{1,2}$ Takahiro Tanaka, ${ }^{3}$ Mayu Tanaka, ${ }^{4}$ and Toshiya Kuno ${ }^{2}$ \\ ${ }^{1}$ The Tohkai Institute of Cytopathology: Cancer Research and Prevention, 5-1-2 Minami-uzura, Gifu City, Gifu 500-8285, Japan \\ ${ }^{2}$ Department of Tumor Pathology, Graduate School of Medicine, Gifu University, Gifu City, Gifu 501-1194, Japan \\ ${ }^{3}$ Department of Physical Therapy, Kansai University of Health Sciences, 2-11-1 Wakaba, Kumatori-Machi, Sennan-Gun, \\ Osaka 590-0482, Japan \\ ${ }^{4}$ Department of Pharmacy, Kinjo Gakuin University, 2-1723 Ohmori, Moriyama-Ku, Nagoya City, Aichi 463-8521, Japan
}

Correspondence should be addressed to Takuji Tanaka, takutt@toukaisaibou.co.jp

Received 26 July 2011; Accepted 29 August 2011

Academic Editor: Masa-Aki Shibata

Copyright ( 2012 Takuji Tanaka et al. This is an open access article distributed under the Creative Commons Attribution License, which permits unrestricted use, distribution, and reproduction in any medium, provided the original work is properly cited.

$\beta$-Cryptoxanthin, a carotenoid, and hesperidin, a flavonoid, possess inhibitory effects on carcinogenesis in several tissues. We recently have prepared a pulp (CHRP) and citrus juices (MJ2 and MJ5) from a satsuma mandarin (Citrus unshiu Mar.) juice (MJ). They contain high amounts of $\beta$-cryptoxanthin and hesperidin. We have demonstrated that CHRP and/or MJs inhibit chemically induced rat colon, rat tongue, and mouse lung tumorigenesis. Gavage with CHRP resulted in an increase of activities of detoxifying enzymes in the liver, colon, and tongue rats. CHRP and MJs were also able to suppress the expression of proinflammatory cytokines and inflammatory enzymes in the target tissues. This paper describes the findings of our in vivo preclinical experiments to develop a strategy for cancer chemoprevention of colon, tongue, and lung neoplasms by use of CHRP and MJs.

\section{Introduction}

Epithelial malignant neoplasms remain a major health challenge in the world. Despite improvements in staging and the application and integration of therapies, including surgery, radiotherapy, and chemotherapy, the 5-year survival rate for individuals with malignancies is still low. Even if strategies for early detection are successful and malignancies are detected at a stage where local tumor resection and treatment is curative, the patients will still have significant risk for developing second primary malignancy associated with the problem, including "field cancerization" [1-6]. For this reason, it is important to focus on chemopreventive strategies to prevent the development of epithelial malignancies $[5,6]$.

Cancer prevention is a rapidly expanding discipline that focuses on the discovery and identification of dietary agents and drugs that prevent or inhibit epithelial malignant tumor development [5-8]. Since approximately one-third of the overall risk of cancer is attributable to diet, a large number of dietary compounds have been tested to determine their chemopreventive ability using animal carcinogenesis models
[4, 9-12]. It is known that certain carotenoids and flavonoids can inhibit cancer development in animal carcinogenesis models $[4,10-12]$. $\beta$-Cryptoxanthin, (Figure 1(a)), a carotenoid, and hesperidin (Figure 1(b)), a flavonoid, are such compounds. $\beta$-Cryptoxanthin with nonsubstituted $\beta$-ionone cycles and provitamin A property possesses several biological activities including scavenging of free radicals, enhancement of gap junctions, immunomodulation, and regulation of enzyme activity involved in carcinogenesis $[13,14]$. $\beta$-Cryptoxanthin is reported to inhibit mouse skin tumorigenesis [15] and rat colon carcinogenesis [16]. Hesperidin, present in several vegetables and fruits, has antioxidant property, antiinflammatory effect, and inhibiting effect on prostaglandin biosynthesis. This flavonoid inhibits chemically induced carcinogenesis in several organs $[4,10-12]$. $\beta$-Cryptoxanthin and hesperidin are thus considered to be potential cancer chemopreventive compounds. However, edible plants contain only small amounts of these chemicals. To obtain higher contents of these compounds in foods, therefore, we prepared a pulp (CHRP) containing high amounts of $\beta$-cryptoxanthin and hesperidin during the process of making satsuma 


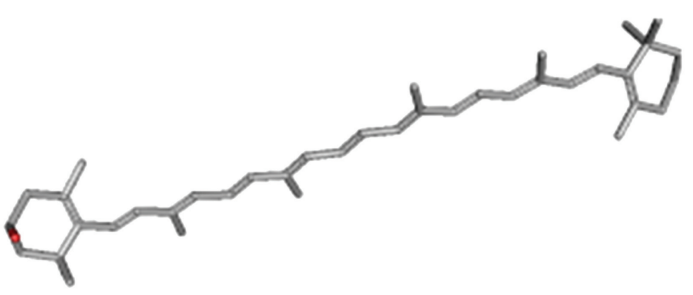

(a)

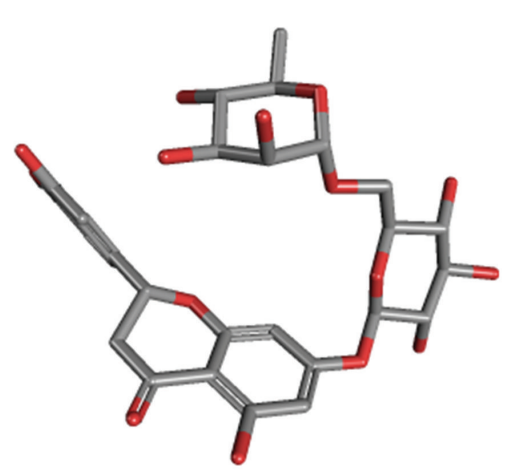

(b)

Figure 1: 3D chemical structures of (a) $\beta$-cryptoxanthin and (b) hesperidin.

mandarin (Citrus unshiu Marc.) juice (MJ). CHRP (100 g) contained $0.67 \mathrm{~g} \beta$-cryptoxanthin and $3.58 \mathrm{~g}$ hesperidin: the contents of $\beta$-cryptoxanthin and hesperidin are 583 times and 38 times greater than those in edible parts of satsuma mandarin (Citrus unshiu Marc.), respectively. In addition, we prepared Satsuma mandarin (Citrus unshiu Marc.) juices, called MJ2 (1.7 mg $\beta$-cryptoxanthin and $84 \mathrm{mg}$ hesperidin/100 g) and MJ5 (84 mg $\beta$-cryptoxanthin and $100 \mathrm{mg}$ hesperidin/100 g), by adding CHRP to usual mandarin orange juice (MJ: $0.8 \mathrm{mg} \beta$-cryptoxanthin and $79 \mathrm{mg}$ hesperi$\operatorname{din} / 100 \mathrm{~g})$.

We describe the chemopreventive effects of CHRP and MJs on chemically induced oncogenesis in the colon and tongue of rats and mouse lung [17-19].

\section{Dietary CHRP Inhibits Chemically Induced Rat Colon Carcinogenesis}

To predict possible inhibitory action of CHRP in colon carcinogenesis, the effects of CHRP on the development of aberrant crypt foci (ACF), which are putative precursor lesions of colonic adenocarcinoma (ADC) of rodents and human [2022], were examined in rats initiated with a colon carcinogen, azoxymethane (AOM) [17]. A total of 32 male F344 rats were used in the study. Animals were divided into the AOM alone, AOM and 500 ppm HCRP, 500 ppm HCRP, and untreated groups. Rats were initiated with AOM by two weekly subcutaneous injections $(20 \mathrm{mg} / \mathrm{kg} \mathrm{bw})$ and were fed the diets containing CHRP at 500 ppm for 4 weeks (initiation stage), starting one week before the first dose of AOM. At week

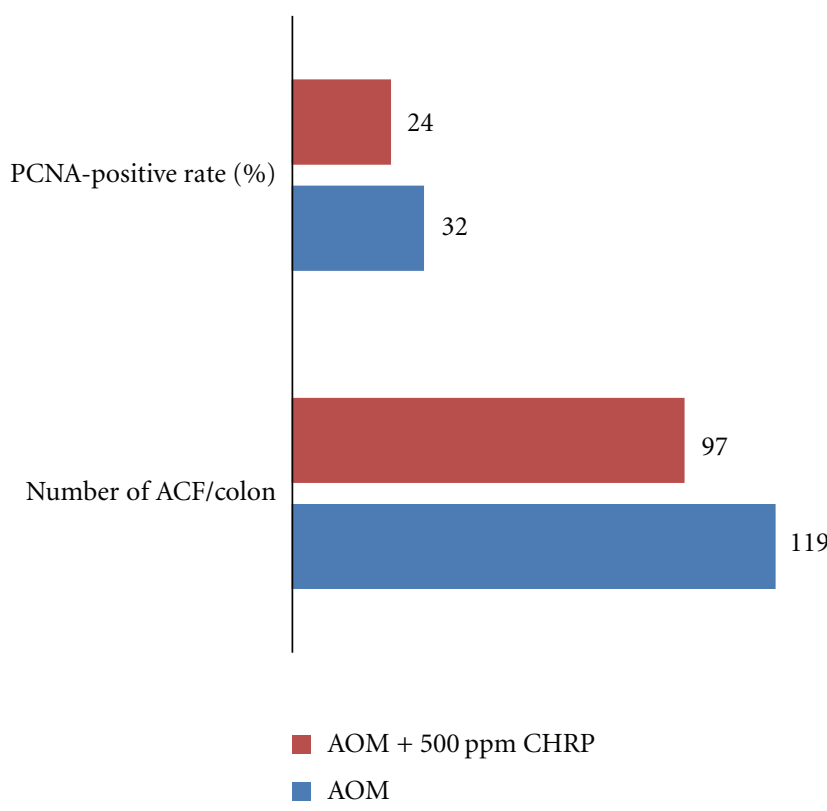

Figure 2: Dietary CHRP suppresses AOM-induced colonic aberrant crypt foci $(\mathrm{ACF})$ in rats.

11 of the experimental week, the frequency of ACF was determined. As illustrated in Figure 2, all rats in the AOMalone group developed ACF with a frequency of $119 \pm 5$ $\mathrm{ACF}$ per rat, while rats in the $\mathrm{AOM}+500 \mathrm{ppm}$ CHRP group had a frequency of $97 \pm 11(P<0.05)$. The CHRP-alone and untreated groups did not develop ACF. Immunohistochemical analysis of proliferation activity, proliferating-cellnuclear-antigen- (PCNA-) positive index, was done, since promising chemopreventive compounds act through modulation of cell proliferative activity in the target organs [11, 23]. As presented in Figure 2, the PCNA-labeling index in ACF was significantly decreased by feeding with the CHIRP $\operatorname{diet}(P<0.05)$.

Based on the findings from this preliminary experiment, a long-term animal experiment was conducted to determine the suppressing effects of CHRP on the development of colonic ADC induced by AOM. A total of 69 rats were randomly divided into 5 groups, namely, the AOM alone, $\mathrm{AOM}+500 \mathrm{ppm}$ CHRP for 4 weeks of the initiation stage, $\mathrm{AOM} \rightarrow 500 \mathrm{ppm} \mathrm{CHRP}$, for 28 weeks of the promotion stage, 500 ppm CHRP for the entire experimental period (32 weeks), and untreated groups. Animals were initiated with three weekly subcutaneous injections of AOM ( $15 \mathrm{mg} / \mathrm{kg} \mathrm{bw})$ to induce colonic neoplasms. As given in Figure 3, the frequencies of colonic ADC in the AOM + 500 ppm CHRP group $(47 \%)$ and the $\mathrm{AOM} \rightarrow 500 \mathrm{ppm}$ CHRP group $(21 \%$, $P<0.05)$ were smaller than in group $1(60 \%)$. In Figure 3, the PCNA-labeling indices of large bowel ADC developed in the the $\mathrm{AOM}+500 \mathrm{ppm} \mathrm{CHRP}$ group and the AOM $\rightarrow$ 500 ppm CHRP group were significantly smaller than the AOM alone group $(P<0.05)$. 


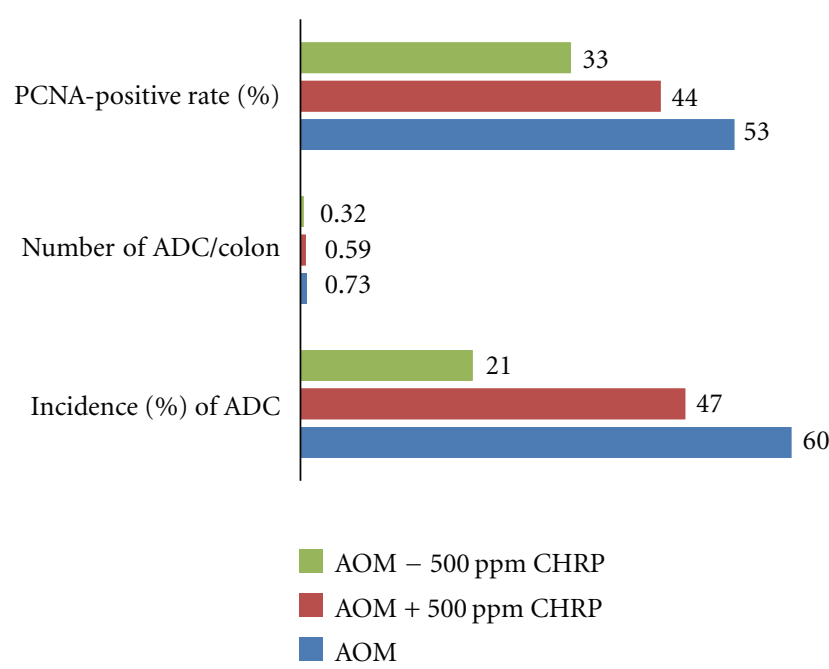

FIgure 3: Dietary CHRP inhibits AOM-induced colonic adenocarcinoma $(\mathrm{ADC})$ in rats.

\section{Supplementation with CHRP in Diet Inhibits 4-Nitroquinoline-1-Oxide- (4-NQO-) Induced Rat Tongue Carcinogenesis}

Oral cancer is an important public health issue because its occurrence is strongly associated with cigarette smoking and alcohol drinking $[2,3,24]$. Oral cancer is one of the ten most frequent cancers worldwide, with three-quarter of all cases occurring in the developing countries $[2,3]$. It varies in the frequency greatly among different countries and geographic regions $[2,3]$. The incidence and mortality of oral cancer have increased over the past decades in Europe [25] and in the United States [26]. In particular, the incidence and mortality rate of tongue cancer, as compared to other types of oral cancer, have increased in younger adults [27]. Chemoprevention against oral/tongue cancer development is thus important. To determine whether CHRP can inhibit chemically induced rat tongue carcinogenesis, a total of 67 male F344 rats were divided into 5 groups, including the 4 -NQO alone group (8-week treatment with $20 \mathrm{ppm} 4$ NQO in drinking water), 4-NQO + 500 ppm CHRP group (10-week treatment during the initiation stage), 4-NQO $\rightarrow 500 \mathrm{ppm}$ CHRP group (22-week treatment during the promotion stage), CHRP alone group (32-week of the entire experiment), and untreated group. At week 32, tongue squamous cell tumors (papilloma and carcinoma) and dysplasia developed in the posterior tongue (dorsal region) of rats that received 4-NQO. As shown in Figure 4 the incidences of tongue squamous cell carcinoma (SCC) were 53\% in the 4 -NQO alone group, $35 \%$ in the $4-\mathrm{NQO}+500 \mathrm{ppm}$ CHRP group, and $5 \%$ in the $4-\mathrm{NQO} \rightarrow 500 \mathrm{ppm} \mathrm{CHRP}$ group. The incidence of tongue carcinoma in the 4-NQO $\rightarrow 500 \mathrm{ppm}$ CHRP group was significantly smaller than the 4-NQOO alone group $(P<0.05)$. The multiplicity of tongue carcinoma of this group was also lower than the 4NQO alone group $(P<0.05)$. The PCNA-labeling index of tongue SCC in the 4 -NQO $\rightarrow 500 \mathrm{ppm}$ CHRP group

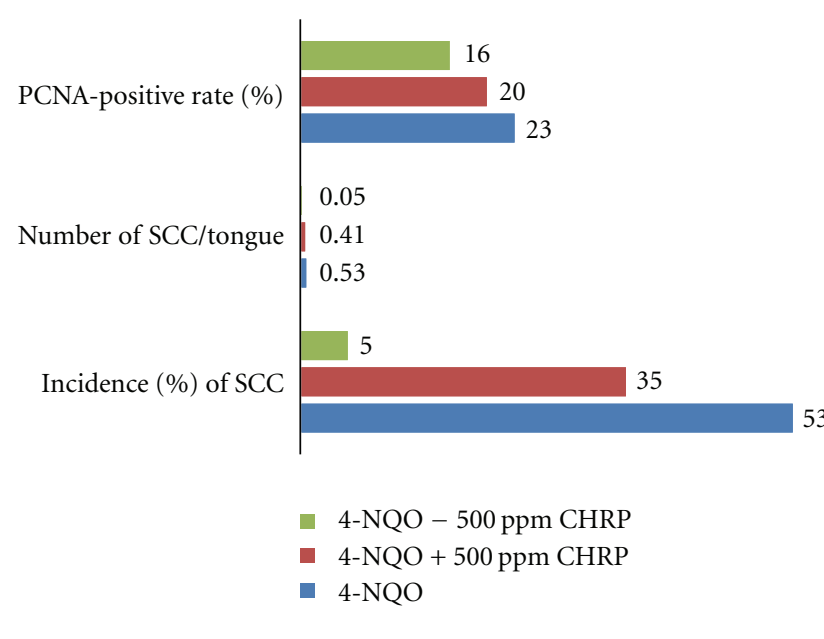

Figure 4: CHRP in diet suppresses 4-NQO-induced rat tongue squamous cell carcinoma (SCC).

was significantly smaller than the 4-NQO alone group $(P<$ $0.05)$.

\section{MJs, Citrus Juices Rich in $\beta$-Cryptoxanthin and Hesperidin, Inhibit AOM-Induced Rat Colon Carcinogenesis}

Colorectal cancer (CRC) is the fourth most common malignant neoplasm in the world [21]. Since an inverse relationship between the intake of fruits/vegetables and human CRC has been suggested [5, 6, 28-32], primary prevention, including chemoprevention utilizing the active compounds in edible plants, is important for reducing this malignancy $[5,6]$. This experiment was designed to determine the modulatory effects of MJ, MJ2, and MJ5 on the occurrence of colonic neoplasms induced by AOM in rats [19]. Also, PCNA-labeling index in colonic neoplasms was analyzed immunohistochemically. A total of 113 male F344 rats were divided into 6 groups: the AOM alone group, the AOM $\rightarrow$ $\mathrm{MJ}$ group, the AOM $\rightarrow$ MJ2 group, the AOM $\rightarrow$ MJ5 group, the MJ5 alone group, and the untreated group. AOM was given to rat by twice weekly subcutaneous injections at a dose level of $20 \mathrm{mg} / \mathrm{kg}$ bw. MJ, MJ2, and MJ5 in black bottles were given to rats for $12 \mathrm{~h}$ (from 8:00 p.m. to 8:00 a.m.). Figure 5 shows the incidence and multiplicity of colonic ADC at week 38. AOM administration induced large intestinal ADC with an incidence of $69 \%$ and a multiplicity of $0.76 \pm 0.57$. The incidences and multiplicities of the $\mathrm{AOM} \rightarrow \mathrm{MJ}$ group, the $\mathrm{AOM} \rightarrow \mathrm{MJ} 2$ group, and the AOM $\rightarrow$ MJ5 group were significantly smaller than the AOM alone group $(P<0.05)$. The mean PCNA-labeling index of adenocarcinoma in rats of group 1 was $55 \pm 7 \%(n=20)$. The mean PCNAlabeling indices of adenocarcinomas present in the AOM $\rightarrow$ MJ2 and the AOM $\rightarrow$ MJ5 groups $(P<0.05)$ were significantly lower than the AOM alone group $(55 \pm 7 \%$, Figure 5). 


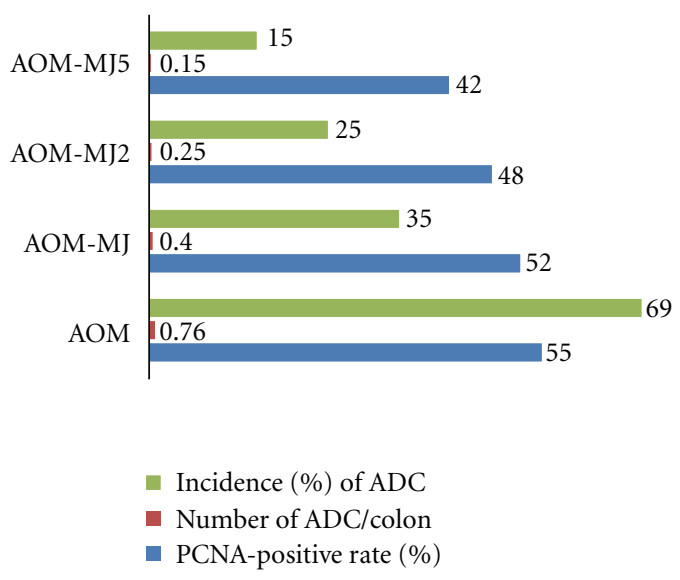

FIgURE 5: MJs suppress AOM-induced colonic adenocarcinoma (ADCs) in rats.

\section{MJs, Citrus Juices Rich in $\beta$-Cryptoxanthin and Hesperidin, Inhibit Chemically Induced Mouse Lung Tumorigenesis}

Lung cancer is the largest cause of cancer deaths in industrial countries, and cigarette smoking is regarded as the overwhelming cause of lung cancer. Chemoprevention using naturally occurring or synthetic compounds to arrest or reverse the carcinogenic process is extremely important as a lung cancer prevention strategy. 4-(Methylnitrosamino)1-(3-pyridyl)-1-butanone (NNK) is the most important carcinogenic nitrosamine, because of its strong potential in inducing lung neoplasms in rodents and its exposure to humans through smoking $[33,34]$. Using the animal model for lung tumorigenesis with NNK, several promising agents for prevention of lung cancers have been reported [33-38]. This experiment was aimed to determine possible modulatory effects of MJs on the development of lung tumors induced by a pulmonary carcinogen, NNK, in mice [18]. The PCNA-labeling index in lung tumors was also determined. A total of 103 male A/J mice were divided into 6 groups: the NNK alone group, the NNK $\rightarrow$ MJ group, the NNK $\rightarrow$ MJ2 group, the NNK $\rightarrow$ MJ5 group, the MJ5 alone group, and the untreated group. NNK was given to mice by a single intraperitoneal injection (10 $\mu \mathrm{mol}$ in saline/mouse). MJ, MJ2, and MJ5 were administered to mice as a drinking water for 21 weeks, starting one week after the NNK injection. MJs in the black bottles were given to mice for $12 \mathrm{hr}$ (from 8:00 p.m. to 8:00 a.m.) At week 22, lung proliferative lesions were diagnosed as hyperplasia and tumors, and we did not subclassify the tumors into adenoma and adenocarcinoma, because of the difficulty in evaluating malignancy $[39,40]$. Pulmonary tumors (adenoma or adenocarcinoma) were developed in all mice treated with NNK. As illustrated in Figure 6, the incidences and the multiplicities of lung tumor of the $\mathrm{NNK} \rightarrow \mathrm{MJ}$ group, the NNK $\rightarrow$ MJ2 group, and the NNK $\rightarrow$ MJ5 group were smaller than NNK alone group. Statistically, the incidence of lung tumors in mice that received NNK and MJ5 was significantly lower than the NNK alone group $(P<0.05)$. The multiplicities of lung

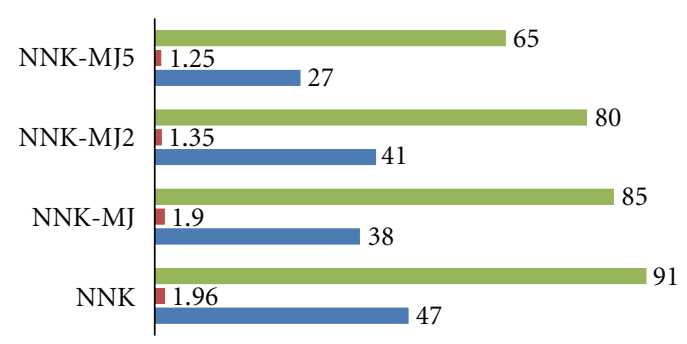

- Incidence (\%) of lung tumors

- Number of tumors/lung

- PCNA-positive rate (\%)

FIgURE 6: MJs inhibit NNK-induced lung tumors in mice.

tumors of mice in the $\mathrm{NNK} \rightarrow \mathrm{MJ}$ group, the $\mathrm{NNK} \rightarrow$ MJ2 group, and the NNK $\rightarrow$ MJ5 group were also low, but the differences were insignificant. The mean PCNA-labeling indices of pulmonary tumors of the NNK $\rightarrow$ MJ group and the NNK $\rightarrow$ MJ5 group were significantly lower than the NNK alone group $(P<0.05$, Figure 6$)$.

\section{CHRP Increases Detoxifying Enzymes in the Liver, Tongue, and Colon}

Induction of enzymes that enhance the detoxification of chemical carcinogens has been a broadly effective strategy for chemoprevention of experimental carcinogenesis in rodent models. Several inducing agents are now in clinical trials to evaluate utility for prevention of cancers associated with unavoidable high exposures to environmental carcinogens. Thus, certain phase II detoxifying enzyme inducers, including phenolic antioxidants, dithiolethiones, isothiocyanates, and triterpenoids, are considered to be promising chemopreventive in preclinical and clinical interventions [41-46].

To determine whether CHRP modifies glutathione $S$ tansferase (GST) and quinone reductase (QR) activities in the liver, colon, and tongue, male F344 rats were gavaged with CHRP at four dose levels $(0,40,200$ or $400 \mathrm{mg} / \mathrm{kg}$ body wt in $0.5 \mathrm{~mL}$ of $5 \%$ gum Arabic) of CHRP for 5 consecutive days [17]. Thirty min after the last gavage, the liver, colon, and tongue were excised immediately to measure GST and QR activities. Dosing of 40, 200, and $400 \mathrm{mg} / \mathrm{kg}$ bw of CHRP significantly elevated liver GST (1.27-fold, 1.25-fold, and 1.50-fold increases, resp., $P<0.05)$ and $\mathrm{QR}$ activities (1.24fold, 1.22-fold, and 1.33-fold increases, resp., $P<0.05$ ) when compared to rats that received $0 \mathrm{mg} / \mathrm{kg}$ bw. Similarly, gavage with CHRP significantly increased GST activity in the colonic (1.11-fold, 1.12-fold, and 1.15-fold increases, resp., $P<0.05)$ and tongue mucosa (1.33-fold, 1.29-fold, and 1.23fold increases, resp., $P<0.05)$. CHRP treatment significantly increased the colonic QR activity at a dose of $400 \mathrm{mg} / \mathrm{kg}$ bw (1.13-fold increase, $P<0.05$ ) and the tongue QR activity at doses of 200 and $400 \mathrm{mg} / \mathrm{kg}$ bw (1.25-fold and 1.21-fold increases, resp., $P<0.05$ ). 


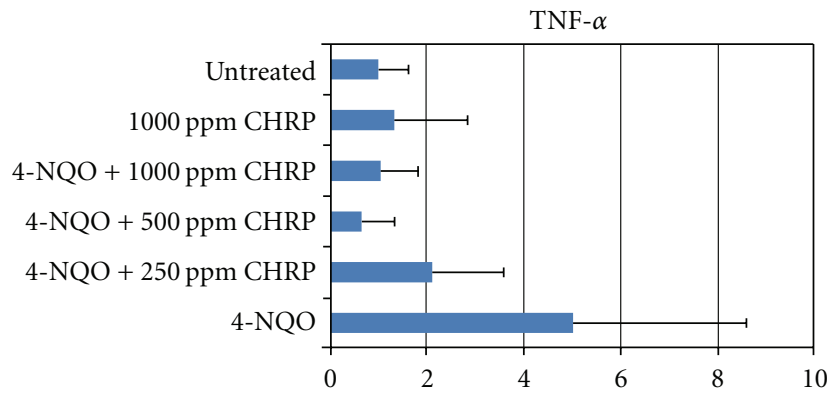

(a)

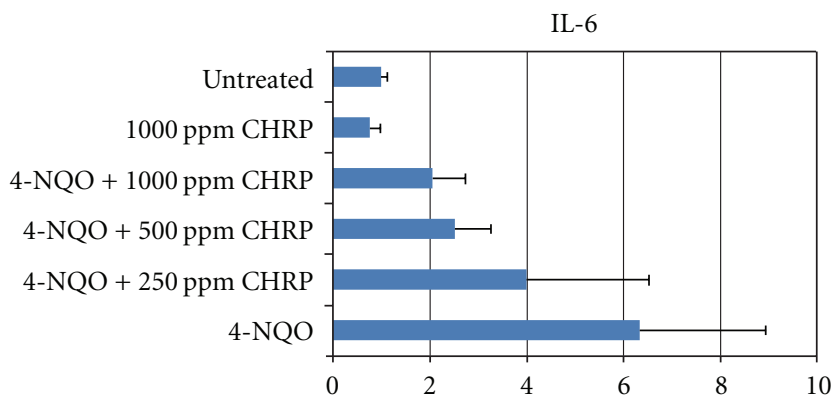

(c)

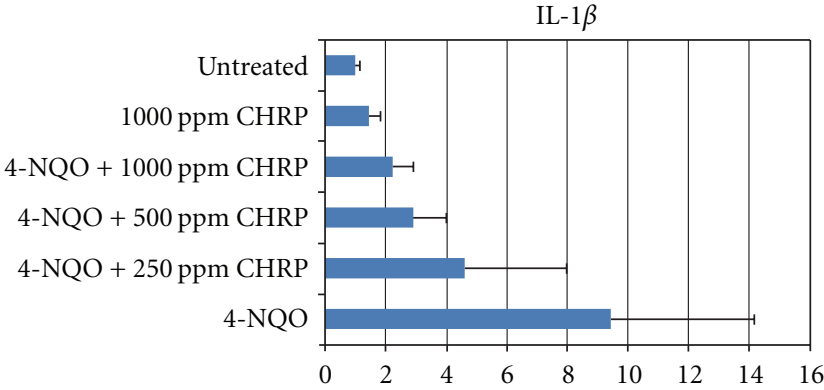

(b)

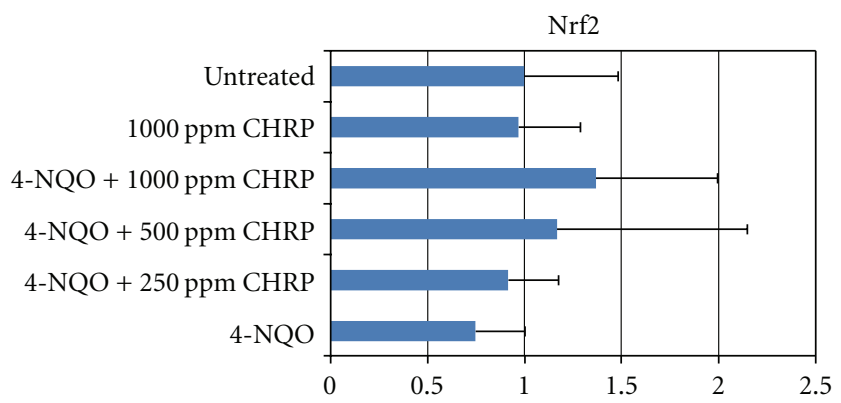

(d)

FIGURE 7: Dietary CHRP lowers mRNA expression of proinflammatory cytokines in the tongue of rats that received 4-NQO at week 8 of the study.

\section{Modulation of Cytokine Expression by CHRP and MJs in the Tongue and Colon of Rats Initiated with Carcinogens}

Chronic inflammation is closely associated with cancer development [47]. Cytokines are an important group of proteins that regulate and mediate inflammation and angiogenesis. Deregulation in their production results in tumor growth, invasion, and metastasis being facilitated. In addition to proinflammatory cytokines, inflammatory enzymes, such as cyclooxygenase (COX)-2 and inducible nitric oxide synthase (iNOS), are involved in carcinogenesis [48-50], and they are good targets for cancer chemoprevention [5154]. The NF-E2-related factor 2 (Nrf2) is a key regulator of the inducible expression of enzymes such as GST and QR in catalyzing the detoxification of reactive electrophiles and oxidants that contribute to the formation of mutations and ultimately cancers. Nrf2 is now recognized to regulate a broad cytoprotective, transcriptional response leading to prevention of damage to DNA. Nrf2 is also a good target for cancer chemoprevention in certain tissues [55-59].

Based on these reports, we assayed mRNA expression of tumor necrosis factor (TNF) $-\alpha$, interleukin (IL) $1-\beta$, IL-6, COX-2, iNOS, and Nrf2 in the tongue and colonic mucosa of rats to determine whether CHRP and MJs can affect this mRNA expression. Male F344 rats were initiated with a tongue carcinogen 4-NQO (20 ppm) by giving drinking water for 8 weeks, and they were also fed diets containing CHRP at doses of 250, 500, and 1000 ppm for 8 weeks, starting 4-NQO administration. At week 8 , tongue mucosa was scraped. In a different experiment, male F344 rats were initiated with a colonic carcinogen AOM by twice weekly subcutaneous injections ( $20 \mathrm{mg} / \mathrm{kg} \mathrm{bw})$, and they also received MJ, MJ2, and MJ5 for 4 weeks from starting the AOM injection. Colonic mucosa was scraped at week 4 of the experimental period. Total RNA was extracted from tongue and colonic mucosa using the RNeasy Mini Kit (Qiagen, Tokyo, Japan) according to the manufacturer's protocol. The cDNA was then synthesized from total RNA using the HighCapacity cDNA Reverse Transcription Kit (Applied Biosystems Japan Ltd, Tokyo, Japan). Quantitative real-time PCR analysis of individual cDNA was performed with ABI Prism 7500 (Applied Biosystems Japan Ltd, Tokyo, Japan) with rat gene specific primers for TNF- $\alpha$, IL- $1 \beta$, IL-6, COX-2, and iNOS (Applied Biosystems) on a GeneAmp 5700 Sequence Detection System (Applied Biosystems). Fold change was calculated using the $\Delta \Delta \mathrm{Ct}$ method relative to untreated with $\beta$-actin as the endogenous control. In addition, the specific primer (forward primer $5^{\prime}$-tgccctggaagtgtcaaa- $3^{\prime}$, reverse $5^{\prime}$-ggctgtactgtatccccagaaga- $3^{\prime}$ ) was used to measure the expression of Nrf2. Data are illustrated in Figures 7, 8, 9, and 10. Administration with CHRP and MJs decreased mRNA expression of TNF- $\alpha$, IL1- $\beta$, IL-6, COX-2, and iNOS in the tongue and colonic mucosa, while the treatments increased mRNA expression of Nrf2 in both mucosal tissues.

\section{Discussion}

We have described cancer chemopreventive ability of CHRP and MJs in experimental animal carcinogenesis models of 


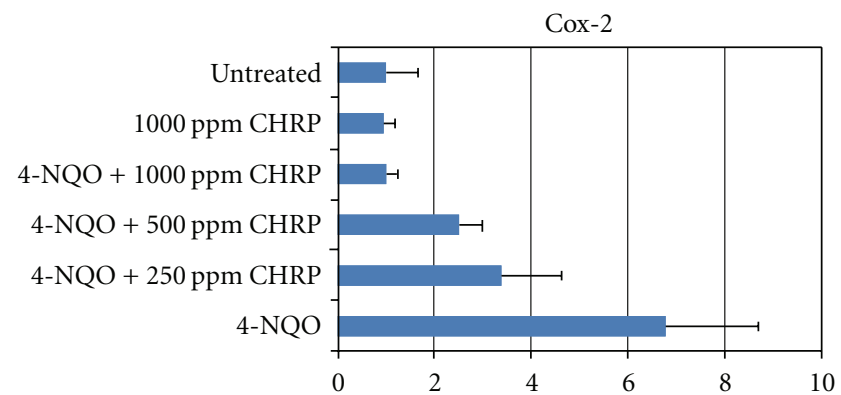

(a)

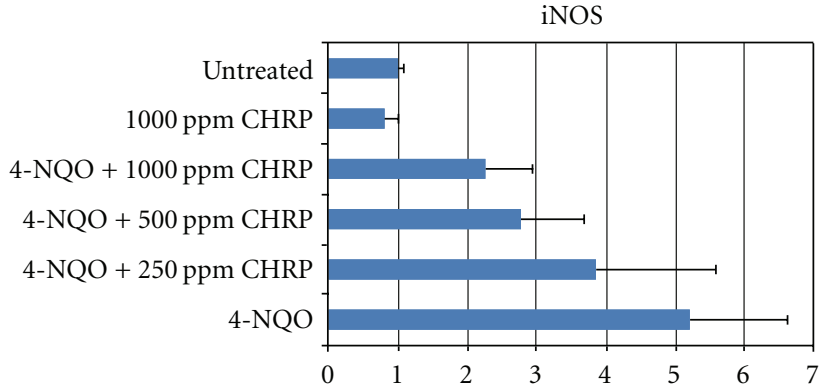

(b)

FIGURE 8: Dietary CHRP suppresses mRNA expression of inflammatory enzymes, Cox-2 and iNOS, in the tongue of rats that received 4-NQO at week 8 of the study.

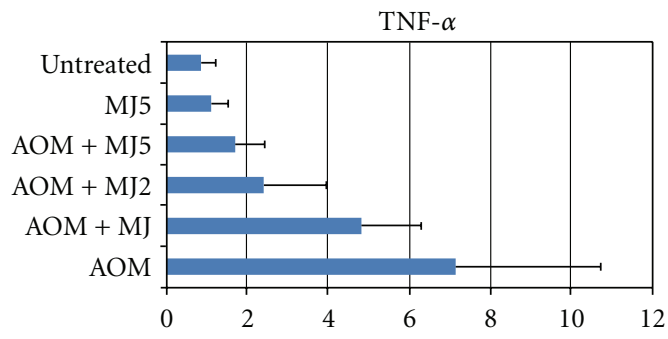

(a)

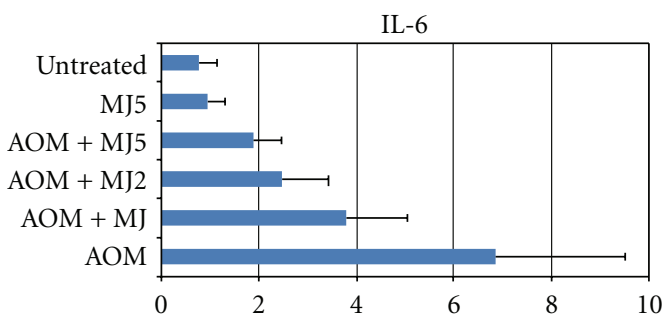

(c)

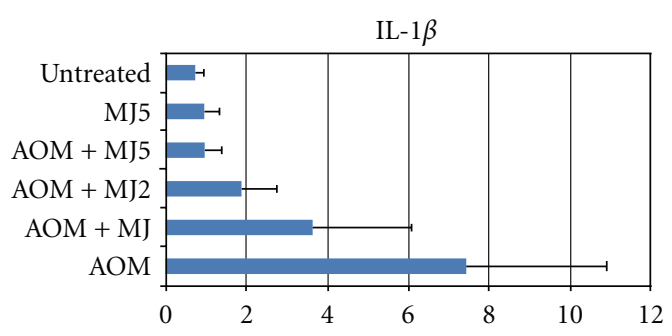

(b)

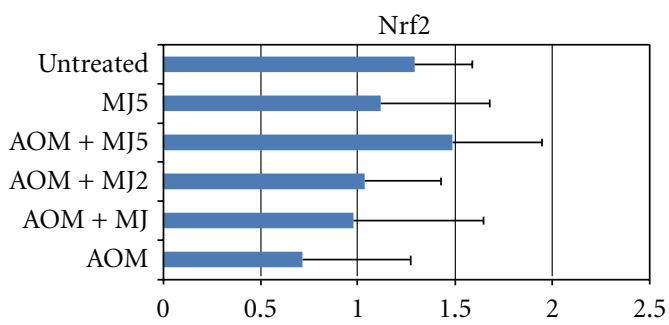

(d)

FIGURE 9: MJs lower mRNA expression of proinflammatory cytokines in the colonic mucosa of rats that received AOM at week 4 of the study.

colon, tongue, and lung cancers. Administration of CHRP or MJs after the carcinogen exposure clearly inhibits the development of malignant epithelial neoplasms. Miyagi et al. [60] also reported a protective effect of orange juice on AOM-induced rat colon carcinogenesis. Our data on MJs support their findings. The exact mechanism(s) involved in the suppressing effects of chemically induced tumorigenesis by CHRP and MJs is not fully known. In our studies, gavage with CHRP to rats elevated GST and QR activities in liver, colon, and tongue. Investigations by other researchers also demonstrated that limonin increased GST activities in several organs including liver, colon, and lung [61]. Therefore, it is possible that such elevation in activity of detoxifying enzyme by CHRP treatment may contribute its chemopreventive ability. Also, the modulatory effects of CHRP and MJs on specific species of liver CYPs, which are pertinent to the carcinogen metabolism $[38,62,63]$, should be considered, although we did not examine their expression in our studies.
In addition, suppressing effects of CHRP and MJs on hypercell-proliferation activity induced by carcinogens in target organs account for their inhibition of carcinogenesis, since control of cell proliferation is one of the important effects of promising chemopreventive agents [23]. In our studies, gavage with CHRP to rats elevated GST and QR activities in liver, colon, and tongue, but strong inhibition in cancer incidence was not found when CHRP was fed to rats during the carcinogen treatment. This indicates that modification of cell proliferation of carcinoma cells rather than modification of activity of detoxifying enzymes may contribute to its chemopreventive ability when the substances were given after carcinogen exposure in long-term experiments.

Interesting findings in our studies are that CHRP and MJs were able to suppress mRNA expression of several cytokines (TNF- $\alpha$, IL-1 $\beta$, IL-6) and inflammatory enzymes (COX-2 and iNOS) and enhance mRNA expression of Nrf2 in the tongue and colon of rats that received a carcinogen, 


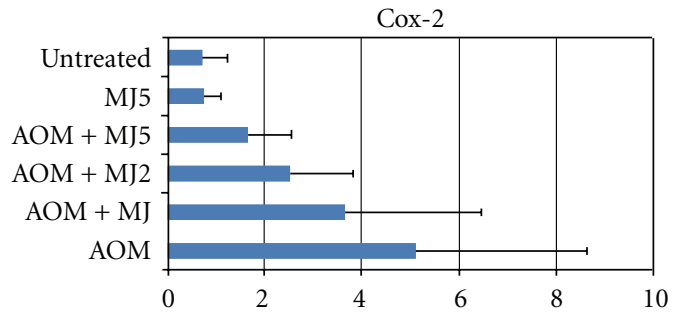

(a)

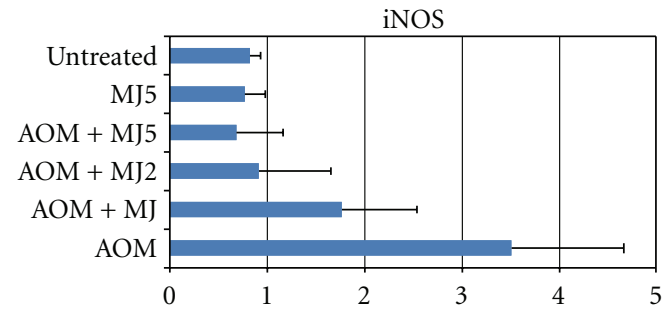

(b)

FIgURE 10: MJs suppress mRNA expression of inflammatory enzymes, Cox-2 and iNOS, in the colonic mucosa of rats that received AOM at week 4 of the study.

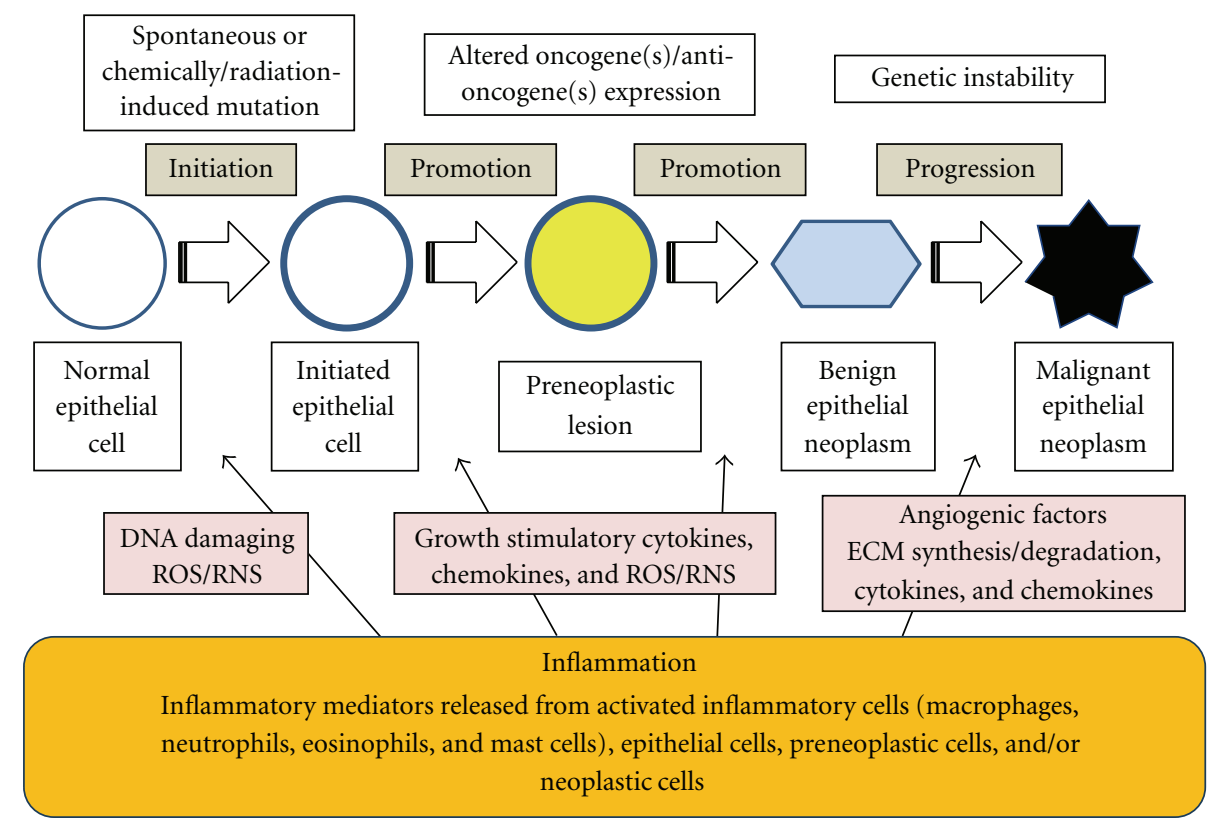

FIGURE 11: Working hypothesis of the involvement of inflammation in multistage carcinogenesis.

4-NQO or AOM. Chronic inflammation is known to be closely associated with cancer development in certain tissues [47], including oral cavity, colon, and lung. Cytokines regulate and mediate inflammation and angiogenesis. Tumor growth, invasion, and metastasis are facilitated when there is a deregulation in their production. Functional polymorphisms affecting gene expression of interleukin IL-1 $\beta, 4,-6$, -8 , and -10 as well as TNF- $\alpha$ are strongly associated with increased risk for oral cancer [64]. Recent findings also indicated that interactions between cytokines and sympathetic neurotransmitters and their respective receptors expressed by the nerve, immune, and tumor cells appear to influence tumor growth [65]. In the colon, inflammatory bowel disease (IBD) is an important risk factor for the development of CRC $[21,66,67]$. Inflammation is also likely to be involved with other forms of sporadic as well as heritable CRC [21]. Relationships between lung inflammation/injury and lung cancer in humans are also suggested [39]. Genetic predisposition to chronic obstructive pulmonary disease was associated with increased risk of developing lung cancer [68]. In addition to proinflammatory cytokines, inflammatory enzymes, such as COX-2 and iNOS, are involved in carcinogenesis [48-50], and they are good targets for cancer chemoprevention [5154]. Nrf2 transcription factor was identified in the mid-1990s as a key regulator of the inducible expression of enzymes such as GST and QR in catalyzing the detoxification of reactive electrophiles and oxidants that contribute to the formation of mutations and ultimately cancers. Nrf2 is now recognized to regulate a broad cytoprotective, transcriptional response leading to prevention of damage to DNA, proteins, and lipids; recognition, repair, and removal of macromolecular damage; and tissue renewal following toxic assaults. The importance of this pathway as a determinant of susceptibility to carcinogenesis was indicated in multiple studies that demonstrated enhanced incidence, multiplicity, and/or tumor burden in Nrf2-deficient mice compared to wild-type ones in models of inflammation and CRC and lung cancer. Nrf2 is thus one of the targets for cancer chemoprevention in certain tissues [55-59]. When considering the relationship between chronic inflammation and cancer development (Figure 11), such effects of CHRP and MJs are attractive for reducing tumor occurrence. 
Citrus fruit contains other possible chemopreventive agents. These include $d$-limonene [69], auraptene [70, 71], diosmin [72], limonin [73], and obacunone [73]. Some of these can modify activities and expression of the detoxifying enzymes and CYPs [74]. In addition, CHRP and MJs are able to downregulate mRNA expression of several cytokines and inflammatory enzymes and upregulate Nrf2 mRNA expression. Thus, citrus fruit is one of the rich sources of cancer chemopreventive agents.

\section{Conclusion}

Our studies demonstrate that oral administration of CHRP and MJs inhibits the development of malignant epithelial neoplasms in colon and tongue and lung tumors of rodents through their multiple biological functions. Further experiments, including preclinical efficacy and mechanistic studies including modification of expression of cancer-related genes in the target tissues, are warranted to fully evaluate these natural compounds for their cancer preventive properties and to understand their mode of action. One of the advantages of CHRP and MJs is that, unlike synthetic chemopreventive agents, they are natural substances present in human foods. On the basis of our observations and knowledge of the carcinogenic process, a strategy for cancer chemoprevention of colon, tongue, and lung can be developed. In conclusion, our findings may indicate that CHRP and MJs that are able to inhibit chemically induced carcinogenesis through modulation of proliferation, detoxifying enzymes, and mRNA expression of several cytokines and inflammatory enzymes are potential cancer chemopreventive agents against tongue, colon, and lung cancer development.

\section{Acknowledgments}

This work was partly supported by a Grant-in-Aid for the 2nd and 3rd Term Comprehensive 10-Year Strategy for Cancer Control, Cancer Prevention, from the Ministry of Health and Welfare of Japan, a Grant-in-Aid for Cancer Research from the Ministry of Health and Welfare of Japan, and a Grant-in-Aid (no. 13671986 and no. 23501324) from the Ministry of Education, Science, Sports and Culture of Japan.

\section{References}

[1] B. E. Johnson, "Second lung cancers in patients after treatment for an initial lung cancer," Journal of the National Cancer Institute, vol. 90, no. 18, pp. 1335-1445, 1998.

[2] T. Tanaka and R. Ishigamori, "Understanding carcinogenesis for fighting oral cancer," Journal of Oncology, vol. 2011, Article ID 603740, 2011.

[3] T. Tanaka, M. Tanaka, and T. Tanaka, "Oral carcinogenesis and oral cancer chemoprevention: a review," Pathology Research International, vol. 2011, Article ID 431246, 2011.

[4] T. Tanaka, "Chemoprevention of oral carcinogenesis," European Journal of Cancer Part B, vol. 31, no. 1, pp. 3-15, 1995.

[5] T. Tanaka, "Chemoprevention of human cancer: biology and therapy," Critical Reviews in Oncology/Hematology, vol. 25, no. 3, pp. 139-174, 1997.
[6] T. Tanaka, "Effect of diet on human carcinogenesis," Critical Reviews in Oncology/Hematology, vol. 25, no. 2, pp. 73-95, 1997.

[7] P. Greenwald, G. J. Kelloff, C. W. Boone, and S. S. McDonald, "Genetic and cellular changes in colorectal cancer: proposed targets of chemopreventive agents," Cancer Epidemiology Biomarkers and Prevention, vol. 4, no. 7, pp. 691-702, 1995.

[8] G. J. Kelloff, C. W. Boone, J. A. Crowell, V. E. Steele, R. Lubet, and C. C. Sigman, "Chemopreventive drug development: perspectives and progress," Cancer Epidemiology Biomarkers and Prevention, vol. 3, no. 1, pp. 85-98, 1994.

[9] D. E. Corpet and S. Taché, "Most effective colon cancer chemopreventive agents in rats: a systematic review of aberrant crypt foci and tumor data, ranked by potency," Nutrition and Cancer, vol. 43, no. 1, pp. 1-21, 2002.

[10] G. J. Kelloff, C. W. Boone, J. A. Crowell et al., "New agents for cancer chemoprevention," Journal of Cellular Biochemistry, vol. 26, supplement, pp. 1-28, 1996.

[11] T. Tanaka and H. Mori, "Inhibition of colon carcinogenesis by non-nutritive constituents in foods," Journal of Toxicologic Pathology, vol. 9, pp. 139-149, 1995.

[12] T. Tanaka and S. Sugie, "Inhibition of colon carcinogenesis by dietary non-nutritive compounds," Journal of Toxicologic Pathology, vol. 20, no. 4, pp. 215-235, 2008.

[13] H. Faure, V. Fayol, C. Galabert et al., "Carotenoids: 1. Metabolism and physiology," Annales de Biologie Clinique, vol. 57, no. 2, pp. 169-183, 1999.

[14] T. Tanaka, H. Sugiura, R. Inaba et al., "Immunomodulatory action of citrus auraptene on macrophage functions and cytokine production of lymphocytes in female BALB/c mice," Carcinogenesis, vol. 20, no. 8, pp. 1471-1476, 1999.

[15] H. Nishino, H. Tokuda, M. Murakoshi et al., "Cancer prevention by natural carotenoids," BioFactors, vol. 13, no. 1-4, pp. 89-94, 2000.

[16] T. Narisawa, Y. Fukaura, S. Oshima, T. Inakuma, M. Yano, and H. Nishino, "Chemoprevention by the oxygenated carotenoid $\beta$-cryptoxanthin of N-methylnitrosourea-induced colon carcinogenesis in F334 rats," Japanese Journal of Cancer Research, vol. 90, no. 10, pp. 1061-1065, 1999.

[17] H. Kohno, M. Maeda, S. Honjo et al., "Prevention of colonic preneoplastic lesions by the beta-cryptoxanthin and hesperidin rich powder prepared from Citrus Unshiu Marc. Juice in male F344 rats," Journal of Toxicologic Pathology, vol. 12, pp. 209-215, 1999.

[18] H. Kohno, M. Taima, T. Sumida, Y. Azuma, H. Ogawa, and T. Tanaka, "Inhibitory effect of mandarin juice rich in $\beta$ cryptoxanthin and hesperidin on 4-(methylnitrosamino)-1(3-pyridyl)-1-butanone-induced pulmonary tumorigenesis in mice," Cancer Letters, vol. 174, no. 2, pp. 141-150, 2001.

[19] T. Tanaka, H. Kohno, M. Murakami et al., "Suppression of azoxymethane-induced colon carcinogenesis in male F344 rats by mandarin juices rich in beta-cryptoxanthin and hesperidin," International Journal of Cancer, vol. 88, pp. 146-150, 2000.

[20] R. P. Bird and C. K. Good, "The significance of aberrant crypt foci in understanding the pathogenesis of colon cancer," Toxicology Letters, vol. 112-113, pp. 395-402, 2000.

[21] T. Tanaka, "Colorectal carcinogenesis: review of human and experimental animal studies," Journal of Carcinogenesis, vol. 8, article 5, 2009.

[22] J. Raju, "Azoxymethane-induced rat aberrant crypt foci: relevance in studying chemoprevention of colon cancer," World Journal of Gastroenterology, vol. 14, no. 43, pp. 6632-6635, 2008. 
[23] H. Mori, S. Sugie, N. Yoshimi, A. Hara, and T. Tanaka, "Control of cell proliferation in cancer prevention," Mutation Research, vol. 428, no. 1-2, pp. 291-298, 1999.

[24] C. Scully, "Oral cancer aetiopathogenesis; past, present and future aspects," Medicina Oral, Pathologia Oral y Cirugia Bucal, vol. 16, no. 3, pp. e306-e311, 2011.

[25] G. J. Macfarlane, L. Sharp, S. Porter, and S. Franceschi, “Trends in survival from cancers of the oral cavity and pharynx in Scotland: a clue as to why the disease is becoming more common?" British Journal of Cancer, vol. 73, no. 6, pp. 805-808, 1996.

[26] C. H. Shiboski, S. C. Shiboski, and S. Silverman, "Trends in oral cancer rates in the United States, 1973-1996," Community Dentistry and Oral Epidemiology, vol. 28, no. 4, pp. 249-256, 2000.

[27] C. La Vecchia, F. Lucchini, E. Negri, P. Boyle, P. Maisonneuve, and F. Levi, "Trends of cancer mortality in Europe, 1955-1989: I, digestive sites," European Journal of Cancer, vol. 28, no. 1, pp. 132-235, 1992.

[28] G. Block, B. Patterson, and A. Subar, "Fruit, vegetables, and cancer prevention: a review of the epidemiological evidence," Nutrition and Cancer, vol. 18, no. 1, pp. 1-29, 1992.

[29] L. O. Dragsted, "Natural antioxidants in chemoprevention," Archives of Toxicology, vol. 20, supplement, pp. 209-226, 1998.

[30] M. Pavia, C. Pileggi, C. G. A. Nobile, and I. F. Angelillo, "Association between fruit and vegetable consumption and oral cancer: a meta-analysis of observational studies," American Journal of Clinical Nutrition, vol. 83, no. 5, pp. 1126-1134, 2006.

[31] E. Riboli and T. Norat, "Epidemiologic evidence of the protective effect of fruit and vegetables on cancer risk," American Journal of Clinical Nutrition, vol. 78, no. 3, supplement, pp. 559S-569S, 2003.

[32] H. Vainio and E. Weiderpass, "Fruit and vegetables in cancer prevention," Nutrition and Cancer, vol. 54, no. 1, pp. 111-142, 2006.

[33] S. S. Hecht and N. Trushin, "DNA and hemoglobin alkylation by 4-(methylnitrosamino)-1-(3-pyridyl)-1-butanone and its major metabolite 4-(methylnitrosamino)-1-(3-pyridyl)-1butanol in F344 rats," Carcinogenesis, vol. 9, no. 9, pp. 1665$1668,1988$.

[34] P. Upadhyaya, P. M. J. Kenney, J. B. Hochalter, M. Wang, and S. S. Hecht, "Tumorigenicity and metabolism of 4-(methylnitrosamino)-1-(3-pyridyl)-1-butanol enantiomers and metabolites in the A/J mouse," Carcinogenesis, vol. 20, no. 8, pp. 1577 $1582,1999$.

[35] G. Akopyan and B. Bonavida, "Understanding tobacco smoke carcinogen NNK and lung tumorigenesis (review)," International Journal of Oncology, vol. 29, no. 4, pp. 745-752, 2006.

[36] H. C. Zheng and Y. Takano, "NNK-induced lung tumors: a review of animal model," Journal of Oncology, vol. 2011, Article ID 635379, 2011.

[37] S. S. Hecht, "Approaches to cancer prevention based on an understanding of N-nitrosamine carcinogenesis," Experimental Biology and Medicine, vol. 216, no. 2, pp. 181-191, 1997.

[38] S. S. Hecht, "Chemoprevention of cancer by isothiocyanates, modifiers of carcinogen metabolism," Journal of Nutrition, vol. 129, no. 3, pp. 768S-774S, 1999.

[39] A. K. Bauer, A. M. Malkinson, and S. R. Kleeberger, "Susceptibility to neoplastic and non-neoplastic pulmonary diseases in mice: genetic similarities," American Journal of Physiology, vol. 287, no. 4, pp. L685-L703, 2004.

[40] W. T. Gunning, A. Castonguay, P. J. Goldblatt, and G. D. Stoner, "Strain A/J mouse lung adenoma growth patterns vary when induced by different carcinogens," Toxicologic Pathology, vol. 19, no. 2, pp. 168-175, 1991.

[41] M. L. Clapper and C. E. Szarka, "Glutathione S-transferasesbiomarkers of cancer risk and chemopreventive response," Chemico-Biological Interactions, vol. 111-112, pp. 377-388, 1998.

[42] A. T. Dinkova-Kostova and P. Talalay, "NAD(P)H:quinone acceptor oxidoreductase 1 (NQO1), a multifunctional antioxidant enzyme and exceptionally versatile cytoprotector," Archives of Biochemistry and Biophysics, vol. 501, no. 1, pp. 116123, 2010.

[43] W. D. Holtzclaw, A. T. Dinkova-Kostova, and P. Talalay, "Protection against electrophile and oxidative stress by induction of phase 2 genes: the quest for the elusive sensor that responds to inducers," Advances in Enzyme Regulation, vol. 44, no. 1, pp. 335-367, 2004.

[44] J. S. Lee and Y. J. Surh, "Nrf2 as a novel molecular target for chemoprevention," Cancer Letters, vol. 224, no. 2, pp. 171-184, 2005.

[45] B. Pool-Zobel, S. Veeriah, and F. D. Böhmer, "Modulation of xenobiotic metabolising enzymes by anticarcinogens focus on glutathione S-transferases and their role as targets of dietary chemoprevention in colorectal carcinogenesis," Mutation Research, vol. 591, no. 1-2, pp. 74-92, 2005.

[46] P. Talalay, "Chemoprotection against cancer by induction of Phase 2 enzymes," BioFactors, vol. 12, no. 1-4, pp. 5-11, 2000.

[47] T. Tanaka and R. Suzuki, "Inflammation and cancer," in Cancer: Disease Progression and Chemoprevention, T. Tanaka, Ed., pp. 27-44, Research Signpost, Kerala, India, 2007.

[48] R. Suzuki, S. Miyamoto, Y. Yasui, S. Sugie, and T. Tanaka, "Global gene expression analysis of the mouse colonic mucosa treated with azoxymethane and dextran sodium sulfate," BMC Cancer, vol. 7, article 84, 2007.

[49] Y. Yasui and T. Tanaka, "Protein expression analysis of inflammation-related colon carcinogenesis," Journal of Carcinogenesis, vol. 8, article 10, 2009.

[50] P. K. Lala and C. Chakraborty, "Role of nitric oxide in carcinogenesis and tumour progression," Lancet Oncology, vol. 2, no. 3, pp. 149-156, 2001.

[51] Y. Yasui, K. Mihe, T. Oyama, and T. Tanaka, "Colorectal car-cin-o-gen-sis and suppression of tumor development by inhibition of enzymes and molecular targets," Current Enzyme Inhibition, vol. 5, no. 1, pp. 1-26, 2009.

[52] S. Guruswamy and C. V. Rao, "Multi-target approaches in colon cancer chemoprevention based on systems biology of tumor cell-signaling," Gene Regulation and Systems Biology, vol. 2, pp. 163-176, 2008.

[53] H. Ohshima, H. Tazawa, B. S. Sylla, and T. Sawa, "Prevention of human cancer by modulation of chronic inflammatory processes," Mutation Research, vol. 591, no. 1-2, pp. 110-122, 2005.

[54] C. V. Rao, "Nitric oxide signaling in colon cancer chemoprevention," Mutation Research, vol. 555, no. 1-2, pp. 107-119, 2004.

[55] R. Hu, C. L. L. Saw, R. Yu, and A. N. T. Kong, "Regulation of NF-E2-related factor 2 signaling for cancer chemoprevention: antioxidant coupled with antiinflammatory," Antioxidants and Redox Signaling, vol. 13, no. 11, pp. 1679-1698, 2010.

[56] J. K. Kundu and Y. J. Surh, "Nrf2-keap1 signaling as a potential target for chemoprevention of inflammation-associated carcinogenesis," Pharmaceutical Research, vol. 27, no. 6, pp. 999 1013, 2010. 
[57] L. Shu, K. L. Cheung, T. O. Khor, C. Chen, and A. N. Kong, "Phytochemicals: cancer chemoprevention and suppression of tumor onset and metastasis," Cancer and Metastasis Reviews, vol. 29, no. 3, pp. 483-502, 2010.

[58] Y. J. Surh, "NF- $\kappa$ B and Nrf2 as potential chemopreventive targets of some anti-inflammatory and antioxidative phytonutrients with anti-inflammatory and antioxidative activities," Asia Pacific Journal of Clinical Nutrition, vol. 17, no. 1, pp. 269272, 2008.

[59] Y. J. Surh, J. K. Kundu, and H. K. Na, "Nrf2 as a master redox switch in turning on the cellular signaling involved in the induction of cytoprotective genes by some chemopreventive phytochemicals," Planta Medica, vol. 74, no. 13, pp. 1526$1539,2008$.

[60] Y. Miyagi, A. S. Om, K. M. Chee, and M. R. Bennink, "Inhibition of azoxymethane-induced colon cancer by orange juice," Nutrition and Cancer, vol. 36, no. 2, pp. 224-229, 2000.

[61] L. K. T. Lam, J. Zhang, S. Hasegawa, and H. A. J. Schut, "Inhibition of chemically induced carcinogenesis by citrus limonoids," in Food Phytochemicals for Cancer Prevention I. Fruits and Vegetables, M. T. Huang, T. Osawa, C. T. Ho, and R. T. Rosen, Eds., pp. 209-219, American Chemical Society, Washington, DC, USA, 1994.

[62] O. S. Sohn, E. S. Fiala, S. P. Requeijo, J. H. Weisburger, and F. J. Gonzalez, "Differential effects of CYP2E1 status on the metabolic activation of the colon carcinogens azoxymethane and methylazoxymethanol," Cancer Research, vol. 61, no. 23, pp. 8435-8440, 2001.

[63] M. D. M. Von Pressentin, K. El-Bayoumy, and J. B. Guttenplan, "Mutagenic activity of 4-nitroquinoline-N-oxide in upper aerodigestive tissue in lacZ mice (Muta(TM)Mouse) and the effects of 1,4- phenylenebis(methylene)selenocyanate," Mutation Research, vol. 466, no. 1, pp. 71-78, 2000.

[64] Z. Serefoglou, C. Yapijakis, E. Nkenke, and E. Vairaktaris, "Genetic association of cytokine DNA polymorphisms with head and neck cancer," Oral Oncology, vol. 44, no. 12, pp. 1093-1099, 2008.

[65] B. Raju and S. O. Ibrahim, "Pathophysiology of oral cancer in experimental animal models: a review with focus on the role of sympathetic nerves," Journal of Oral Pathology and Medicine, vol. 40, no. 1, pp. 1-9, 2011.

[66] T. Tanaka, H. Kohno, M. Murakami, R. Shimada, and S. Kagami, "Colitis-related rat colon carcinogenesis induced by 1-hydroxyanthraquinone and methylazoxymethanol acetate (review)," Oncology Reports, vol. 7, no. 3, pp. 501-508, 2000.

[67] D. W. Rosenberg, C. Giardina, and T. Tanaka, "Mouse models for the study of colon carcinogenesis," Carcinogenesis, vol. 30, no. 2, pp. 183-196, 2009.

[68] B. H. Cohen, E. L. Diamond, C. G. Graves et al., "A common familial component in lung cancer and chronic obstructive pulmonary disease," The Lancet, vol. 2, no. 8037, pp. 523-526, 1977.

[69] T. Kawamori, T. Tanaka, Y. Hirose, M. Ohnishi, and H. Mori, "Inhibitory effects of d-limonene on the development of colonic aberrant crypt foci induced by azoxymethane in F344 rats," Carcinogenesis, vol. 17, no. 2, pp. 369-372, 1996.

[70] T. Tanaka, K. Kawabata, M. Kakumoto et al., "Citrus auraptene exerts dose-dependent chemopreventive activity in rat large bowel tumorigenesis: the inhibition correlates with suppression of cell proliferation and lipid peroxidation and with induction of phase II drug- metabolizing enzymes," Cancer Research, vol. 58, no. 12, pp. 2550-2556, 1998.
[71] T. Tanaka, Y. Yasui, R. Ishigamori-Suzuki, and T. Oyama, "Citrus compounds inhibit inflammation- and obesity-related colon carcinogenesis in mice," Nutrition and Cancer, vol. 60, no. 1, pp. 70-80, 2008.

[72] T. Tanaka, H. Makita, K. Kawabata et al., "Chemoprevention of azoxymethane-induced rat colon carcinogenesis by the naturally occurring flavonoids, diosmin and hesperidin," Carcinogenesis, vol. 18, no. 5, pp. 957-965, 1997.

[73] T. Tanaka, M. Maeda, H. Kohno et al., "Inhibition of azoxymethane-induced colon carcinogenesis in male F344 rats by the citrus limonoids obacunone and limonin," Carcinogenesis, vol. 22, no. 1, pp. 193-198, 2001.

[74] A. Murakami, K. Wada, N. Ueda et al., "In Vitro absorption and metabolism of a citrus chemopreventive agent, auraptene, and its modifying effects on xenobiotic enzyme activities in mouse livers," Nutrition and Cancer, vol. 36, no. 2, pp. 191$199,2000$. 

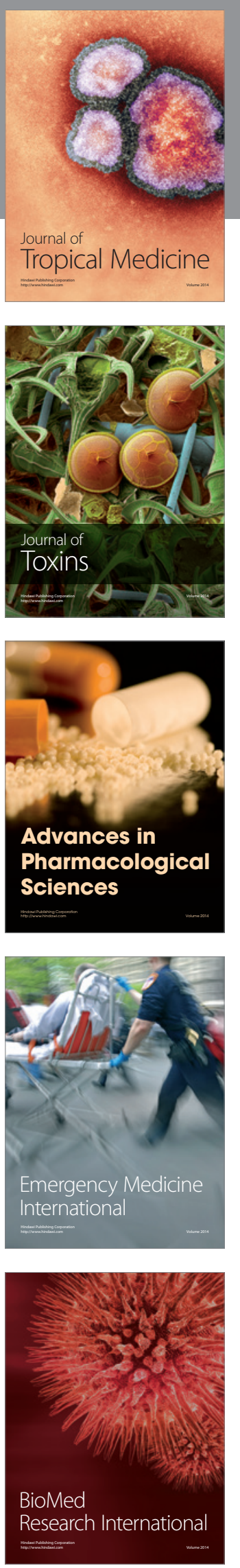
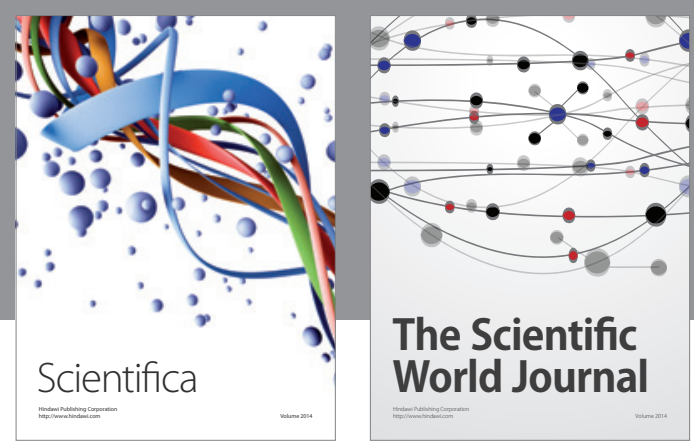

The Scientific World Journal
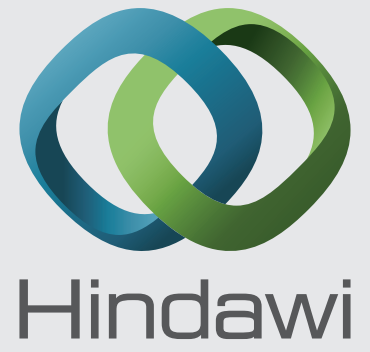

Submit your manuscripts at

http://www.hindawi.com
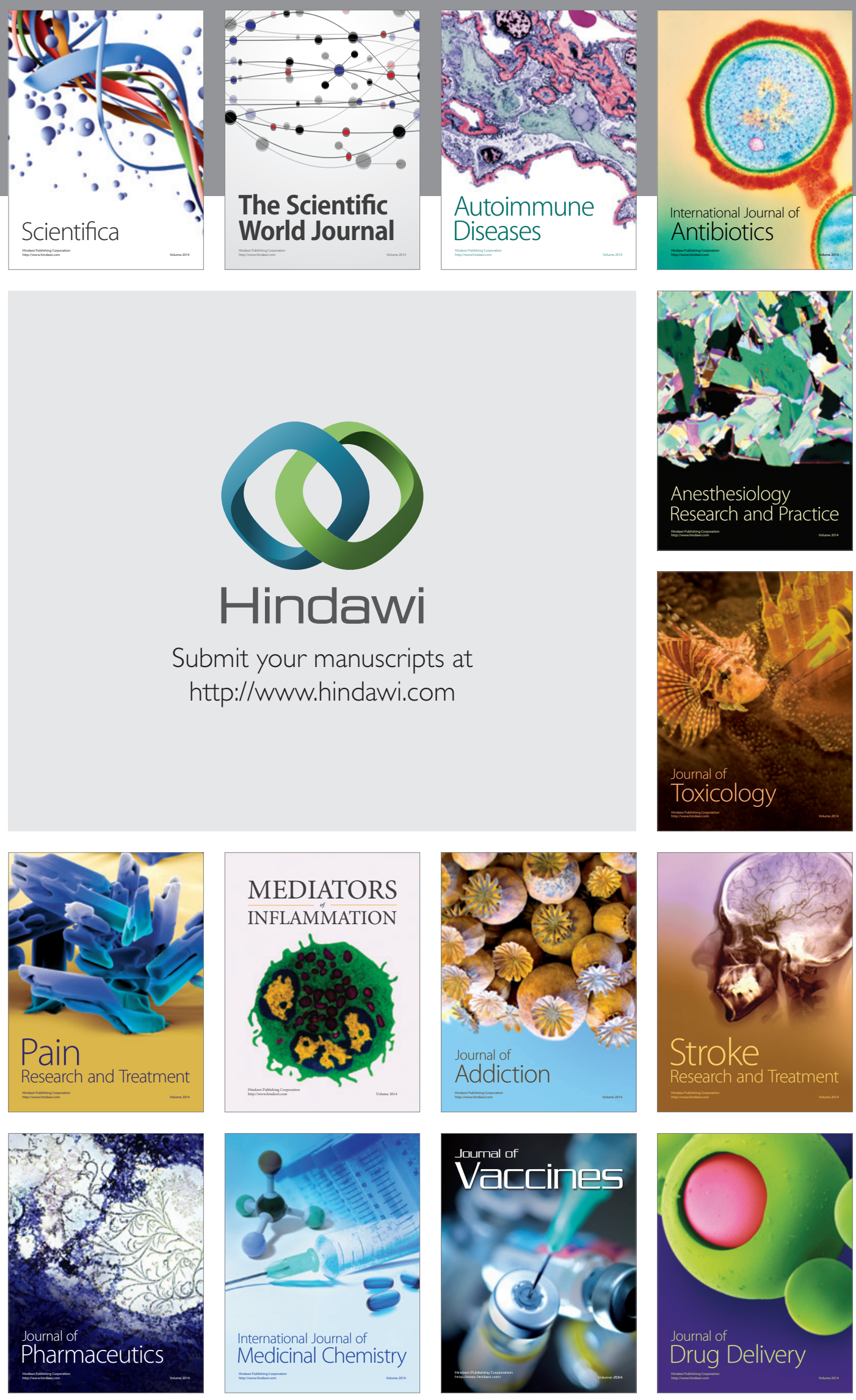